\title{
PROJECTIVE MODULES WITH FREE MULTIPLES AND POWERS
}

\author{
HYMAN BASS AND ROBERT GURALNICK
}

\begin{abstract}
Let $P$ be a finitely generated projective module over a commutative ring.
\end{abstract} Some tensor power of $P$ is free iff some sum of copies of $P$ is free.

Theorem. Let $A$ be a commutative ring and $P$ a finitely generated projective $A$-module of rank $r>0$.

(a) Suppose that $P^{\otimes n}\left(=P \otimes_{A} \cdots \otimes_{A} P\right.$, $n$ times $)$ is free for some integer $n>0$. Then $m P(=P \oplus \cdots \oplus P, m$ times $)$ is free for some integer $h>0$, where $m=$ $r^{h(n-1)} \cdot n^{h}$.

(b) Suppose that $m P$ is free for some integer $m>0$. Then for some integer $t>0$, $P^{\otimes m^{t}}$ is free.

Since $P$ and the assumed isomorphisms with free modules are defined over some finitely generated subring of $A$, there is no loss in assuming, as we do, that $A$ is noetherian, say of dimension $d$.

In $K_{0}(A)$ we put $x=[P]$ and $y=x-r$.

Proof of (a). By hypothesis, $x^{n}=r^{n}$ so

$$
0=x^{n}-r^{n}=y z
$$

where $z=x^{n-1}+x^{n-2} r+\cdots+x r^{n-2}+r^{n-1}$. We have

$$
\begin{aligned}
z-n r^{n-1} & =\sum_{i=1}^{n}\left(x^{n-i} r^{i-1}-r^{n-1}\right) \\
& =\sum_{i=1}^{n} r^{i-1}\left(x^{n-i}-r^{n-i}\right)=-y w
\end{aligned}
$$

for some $w$, whence $n \cdot r^{n-1}=z+y w$. For $h>0$ we thus have

$$
n^{h} r^{h(n-1)}=z u+y^{h} w^{h}
$$

for some $u$. According to [B, Chapter IX, Proposition (4.4)], $y$ is nilpotent, in fact $y^{h+1}=0$ for some $h \leqslant d=\operatorname{dim}(A)$. It follows then from (1) and (2) that, with $m=n^{h} r^{h(n-1)}$, we have $m y=0$, i.e. $m x=m r$, i.e. $[m P]=\left[m A^{r}\right]$. Enlarging $h$, if necessary, so that $m r>d$, it then follows from the Cancellation Theorem [B, Chapter III, Corollary (3.5)], that $m P \cong m A^{r}=A^{m r}$.

Received by the editors February 13, 1985 and, in revised form, April 3, 1985.

1980 Mathematics Subject Classification. Primary 13C10, 13D15.

(C)1986 American Mathematical Society $0002-9939 / 86 \$ 1.00+\$ .25$ per page 
Proof of (b). By assumption, $m x=m r$, i.e. $m y=0$. By induction on $t \geqslant 1$ we can write $x^{m^{t}}=r^{m^{t}}+y^{2^{t}} z_{t}$ for some $z_{t} \in K_{0}(A)$. Indeed $x=r+y$ so $z_{1}=1$. By induction,

$$
\begin{aligned}
x^{m^{t+1}} & =\left(x^{m^{t}}\right)^{m}=\left(r^{m^{t}}+y^{2^{t}} z_{t}\right)^{m} \\
& =\left(r^{m^{t}}\right)^{m}+m y^{2^{t}} z_{t}\left(r^{m^{t}}\right)^{m-1}+\left(y^{2^{t}}\right)^{2} z_{t+1},
\end{aligned}
$$

whence the claim, since $m y=0$. Now as above, $y^{h}=0$ for some $h \leqslant d+1$. Taking $t$ large enough so that $2^{t} \geqslant h$, we then have $x^{m^{t}}=r^{m^{t}}$, i.e. $\left[P^{\otimes m^{t}}\right]=\left[\left(A^{r}\right)^{\otimes m^{t}}\right]=$ [ $A^{r^{m^{\prime}}}$ ]. If $r=1$ then $P^{\otimes m^{\prime}} \cong A$, so assume that $r>1$. With $t$ large enough so that $r^{m^{\prime}}>d=\operatorname{dim}(A)$ we again conclude from the Cancellation Theorem that $P^{\otimes m^{t}}$ is free.

\section{REFERENCES}

[B] H. Bass, Algebraic K-theory, Benjamin, New York, 1968.

Department of Mathematics, Columbia University, New York, New York 10027

Department of Mathematics, University of Southern California, Los Angeles, California 90089 